\title{
Propiedades electrónicas de un anillo cuántico elíptico con sección transversal rectangular (Anillo cuántico elíptico)
}

\author{
$\checkmark$ Juan Alejandro Vinasco SuÁrez ${ }^{1}$ \\ ADRIAN RADU ${ }^{2}$ \\ CARlos Alberto Duque Echeverri ${ }^{1}$
}

\section{Resumen}

Los estados electrónicos de un anillo cuántico elíptico de GaAs embebido en una matriz de $\mathrm{Al}_{\mathrm{x}} \mathrm{Ga}_{1-\mathrm{x}} \mathrm{As}$ son investigados mediante la aproximación de masa efectiva. El anillo cuántico es construido con una sección transversal rectangular (dirección radial). La ecuación de Schrödinger es resuelta mediante el método de elementos finitos. En dirección angular se modula la amplitud de la altura, lo que permite la generación de puntos cuánticos a lo largo del anillo. Se reportan las energías del electrón como función de las dimensiones del anillo, tanto las longitudes de las elipses en el plano $x y$ como su altura (eje $z$ ).

Palabras claves: Anillo cuántico elíptico; Confinamiento finito; Método de elementos finitos; Puntos cuánticos.

\section{Electronic properties of an elliptical quantum ring with rectangular cross section (Elliptical quantum ring)}

\section{Abstract}

The electronic states of a GaAs elliptic quantum ring embedded in $\mathrm{Al}_{\mathrm{x}} \mathrm{Ga}_{1-\mathrm{x}}$ As matrix are investigated by mean of effective mass approximation. The quantum ring is built with rectangular cross section (radial

1 Universidad de Antioquia, Medellín, Colombia.

2 Universidad Politécnica de Bucarest, Bucarest, Rumania.

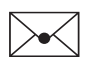

Autor de correspondencia: Vinasco Suárez, J.A. (Juan Alejandro): Grupo de Materia Condensada. Instituto de Física, Facultad de Ciencias Exactas y Naturales, Universidad de Antioquia UdeA, Calle 70 No. 52-21, Medellín, Colombia. Teléfono: (57) 3173610072. Correo electrónico: juan.vinascos@udea.edu.co
Historia del artículo:

Artículo recibido: 9-VIII-2018/ Aprobado: 18-XII-2018

Disponible online: 3 de febrero de 2019

Discusión abierta hasta octubre de 2020 
direction). The Schrödinger equation is solved by using finite element method. In angular direction, the amplitude of altitude is modulated, it lets to model quantum dots in the quantum ring. The electron energies are reported as a function of size ring, for both the lengths of the ellipses in the $x y$ plane and their height ( $z$-axis).

Keywords: Elliptic quantum ring; Finite confinement; Finite element method; Quantum dots.

\section{Propriedades eletrônicas de um anel quântico elíptico com seção transversal retangular (anel quântico elíptico)}

\section{Resumo}

Os estados eletrônicos de um anel quântico elíptico de GaAs embutido em uma matriz de $\mathrm{Al}_{x} \mathrm{Ga}_{1-\mathrm{x}}$ As são investigados pela aproximação da massa efetiva. 0 anel quântico é construído com uma seção transversal retangular (direção radial). A equação de Schrödinger é resolvida pelo método dos elementos finitos. Na direção angular, a amplitude da altura é modulada, o que permite a geração de pontos quânticos ao longo do anel. As energias do elétron são relatadas como uma função das dimensões do anel, tanto os comprimentos das elipses no plano xy e sua altura (eixo z).

Palavras-chave: Anel quântico elíptico; Confinamento finito, Método dos elementos finitos; Pontos quânticos.

\section{Introducción}

El desarrollo en las técnicas de crecimiento para estructuras de baja dimensionalidad ha permitido la construcción de sistemas con variadas geometrías, cuyas propiedades optoelectrónicas son controlables mediante tamaño y forma. Los anillos cuánticos ( $Q R$, por sus siglas en inglés) han sido objeto de diversos estudios, tanto teóricos como experimentales con múltiples efectos, entre los que se incluyen campos eléctricos y magnéticos, impurezas, presión, temperatura y señal de láseres, Hu y Wang (2018); Escorcia, García y Mikhailov (2018); El-Bakkari et al. (2018); Mughnetsyan et al. (2013); Chakraborty et al. (2018); Shi y Yan (2018); Gómez et al. (2009). Desde los desarrollos experimentales existe evidencia de la dependencia de la geometría con la técnica de síntesis usada y las condiciones del crecimiento, Fomin (2014); Lorke et al. (2000); Ling y Lee (2007); Cheng, Yang y Yang (2000). En particular, los QRs son difíciles de obtener con una forma circular perfecta, de ahí que un modelo más realista corresponda a QRs con forma elíptica. Es claro que los QRs con simetría circular son un caso particular de los elípticos, no siendo correcta la opción contraria. De ese modo, considerados el caso elíptico como una muy buena generalización de los QRs cuasi-bidimensionales.

Dentro de las aplicaciones que se pueden encontrar para los QRs están los sensores en el rango del infrarrojo medio y de los $\mathrm{THz}$, como medio activo de los láseres, memorias y dispositivos de la spintrónica, Wu et al. (2012); Mano et al. (2007); Ling et al. (2009); Baker et al. (2007); Lee et al. (2006); Rosas, Riera y Marín (2000).

Para la fabricación de los QRs están las técnicas de Epitaxia de Haces Moleculares y la Deposición Química de Vapores que permiten construir QRs con forma elíptica y en algunos casos con geometrías casi circulares, Fomin (2014). Un QR 
individual o doble ha sido obtenido mediante la técnica epitaxia droplet, P. Boonpeng et al. (2013); Linares-García et al. (2016); Kuroda et al. (2005). Otra técnica usualmente usada es la StranskiKrastanov, Lorke et al. (2003), mediante la cual se obtienen anillos y puntos cuánticos formados por los efectos de tensión que se dan cuando se ensamblan materiales semiconductores que tienen constantes de red suficientemente diferentes. A estos sistemas obtenidos por dicha técnica se les llama anillos y puntos cuánticos autoensamblados.

Los estudios teóricos relacionados con QRs incluyen el efecto Aharonov-Bohm, Aharonov y Bohm (1959), el cual es favorecido por la topología de los QRs, que tiene a su vez importantes implicaciones sobre la corriente persistente del electrón, Büttiker, Imry y Landauer (1983). Mughnetsyan et. Al (2013) estudiaron el efecto de la presión hidrostática y la interacción Rashba spin-órbita en QRs cilíndricos, donde se ha investigado el espectro de energía y la absorción de la luz. El-Bukkari et. al (2018) estudiaron las energías de enlace y de emisión interbanda para un excitón confinado en un QR de GaAs/ $\mathrm{Ga}_{1-x} \mathrm{Al}$ As con efectos de temperatura y presión. En Gómez et al. (2009) investigaron el espectro de energía para QRs con sección transversal circular y

rectangular con efectos de impureza donadora. En Escorcia, García y Mikhailov (2018), la influencia magnetoeléctrica y de impureza no centrada son estudiadas usando el método Kane.

En un reciente estudio realizado por He et. al (2017), se investigaron las propiedades de transporte electrónico mediante la técnica de la función Keldysh Green para QRs formados por tres puntos cuánticos ubicados en los vértices de un triángulo. Bejan, Stan, y Niculescu (2018a) estudiaron propiedades electrónicas y ópticas en QRs elípticos con efectos de campo eléctrico. Recientemente, Vinasco et al. (2017) reportaron la electroabsorción en anillos cuánticos elípticos de GaAs con sección transversal rectangular tenien- do en cuenta efectos de campo eléctrico e impureza donadora.

Mughnetsyan y Kirakosyan (2017) estudiaron la distribución de fuerzas de tensión y la estructura de bandas en una superred de anillos cuánticos de InA/GaAs. En Collier, Saroka y Portnoi (2017), las transiciones son controladas mediante dos hilos cargados en el plano del anillo. En estudios muy recientes, $\mathrm{Hu}$ y Wang (2018) investigaron los efectos de un campo eléctrico externo sobre la energía de enlace de una impureza hidrogénica en dos QRs concéntricos de InGaAsP/InP. Zamani et al. (2018) investigaron los efectos que tienen el campo magnético, la temperatura y las dimensiones de un anillo cuántico de GaAs sobre la transparencia inducida electromagnéticamente (EIT, por sus siglas en inglés). En Bejan, Stan, y Niculescu (2018b) se estudian los efectos de impureza, campo eléctrico, la intensidad y polarización de láseres de control y de prueba sobre la EIT.

En el presente trabajo se estudian las energías y funciones de onda para un electrón confinado en un QR como función del número de puntos cuánticos sobre el anillo, las dimensiones y amplitud del QR. Para la obtención de resultados, la ecuación de Schrödinger es resuelta mediante el método de elementos finitos (MEF) usando el Software COMSOL Multiphysics v. 5.3a. La técnica MEF permite modelar con muy buena precisión diferentes formas de las heteroestructuras considerando su extensión en el espacio tridimensional. Permite también considerar la variación de la masa efectiva con la posición, es decir dentro y fuera de los QRs. No tenemos conocimiento de que en la literatura se hayan reportado estudios similares para un problema como el que aquí se aborda.

El artículo se desglosa así: en la Sección II se presenta un muy breve marco teórico, los resultados y discusión se presentan en la sección III, finalmente las conclusiones son descritas en la sección IV. 


\section{Modelo teórico}

En el presente trabajo se considera un $\mathrm{QR}$ de GaAs sumergido en una matriz de $\mathrm{Al}_{0.3} \mathrm{Ga}_{0.7}$ As. La forma del QR está determinada por una base en forma de corona elíptica y una altura conformada por una superficie diferencial con forma rectangular. La desigualdad que delimita la base del QR, en coordenadas cartesianas está dada por:

$$
g\left(x, y, R x_{2}, R y_{2}\right)<1<f\left(x, y, R x_{1}, R y_{1}\right)
$$

donde $f$ y $g$ están dadas por las siguientes formas elípticas, que representan los bordes interior y exterior del QR:

$$
\begin{aligned}
& f\left(x, y, R x_{1}, R y_{1}\right)=\left(\frac{x}{R x_{1}}\right)^{2}+\left(\frac{y}{R y_{1}}\right)^{2}=1, \\
& g\left(x, y, R x_{2}, R y_{2}\right)=\left(\frac{x}{R x_{2}}\right)^{2}+\left(\frac{y}{R y_{2}}\right)^{2}=1 .
\end{aligned}
$$

Para la sección transversal del QR se han usado funciones trigonométricas para modular la altura $h$ en dirección angular, la cual está dada por:

$$
h(x, y)=H+A \cos (n \theta)
$$

donde $H$ es la altura de referencia del $\mathrm{QR}, A$ corresponde a la amplitud de modulación de la altura, $n=0,1,2$ y 3 es el número de puntos cuánticos sobre el QR y $\theta=\arccos \left(\frac{x}{\sqrt{x^{2}+y^{2}}}\right)$.

El Hamiltoniano 3D para un electrón confinado en el QR en las aproximaciones de masa efectiva y banda de conducción parabólica está dado por:

$$
H=-\frac{\hbar^{2}}{2} \nabla \cdot\left(\frac{1}{m^{*}} \nabla_{x, y, z}\right)+V(x, y, z)
$$

donde los valores de $m *$ y $V(x, y, z)$ son dependientes de la región para la cual la ecuación de Schrödinger es resuelta:

$$
m^{*}= \begin{cases}m_{i}^{*}, & \text { Para el QR (GaAS) } \\ m_{o}^{*}, & \text { Para la matriz (AlGaAs) }\end{cases}
$$

y

$$
V(x, y, z)=\left\{\begin{array}{cl}
0, & \text { Para el QR (GaAS) } \\
V_{o}, & \text { Para la matriz (AlGaAs) }
\end{array}\right.
$$

Los parámetros usados para sistema del QR son: $m_{i}^{*}=0,067 m_{o^{\prime}} m_{0}{ }^{*}=0,092 m_{o}$ [Kuroda et al. (2005)], donde $m_{o}$ es la masa del electrón libre.

Figura 1. Representación del sistema en COMSOL Multiphysics. En (a) anillo de GaAs sumergido en AIGaAs y en (b) la malla correspondiente para el método de elementos finitos.
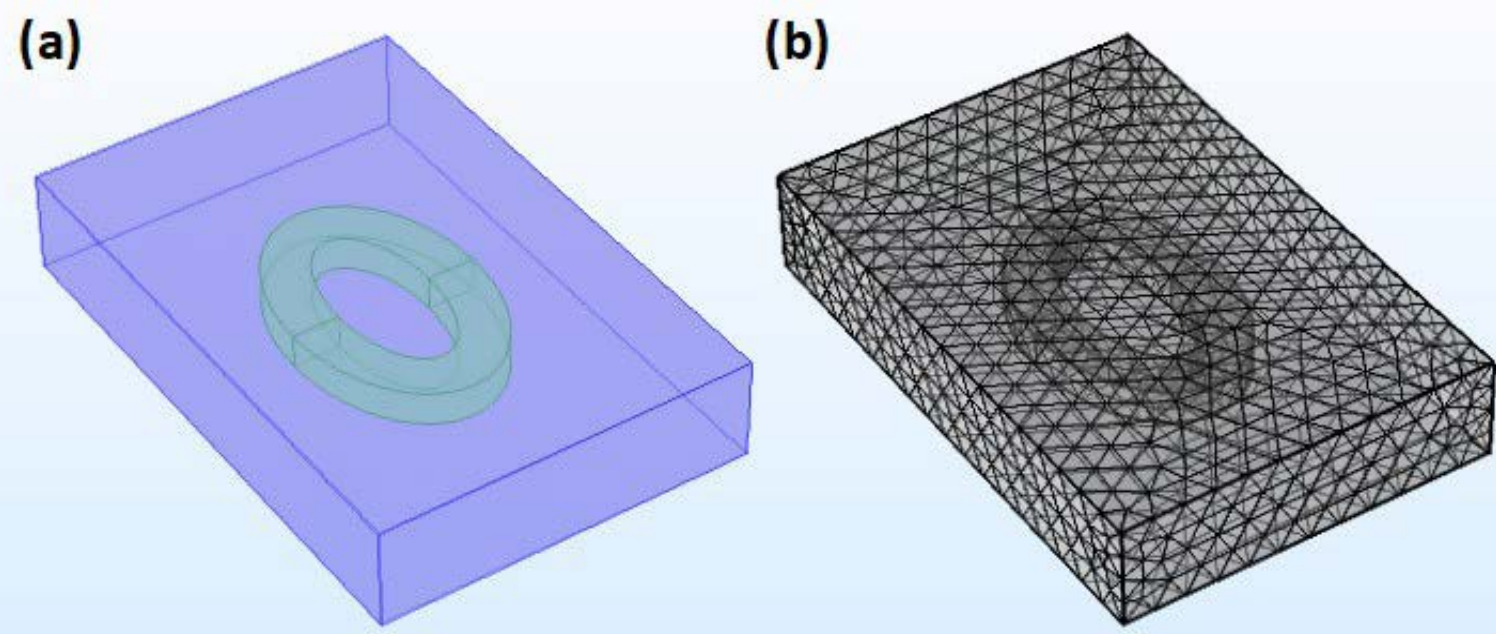
Figura 2. QRs estudiados en este artículo. Diferente número de puntos cuánticos se consideraron $n=0,1,2,3$ [ver Ecuación (4)] para los literales (a-d), respectivamente.

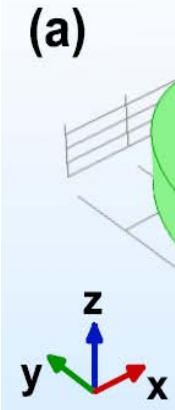

(c)

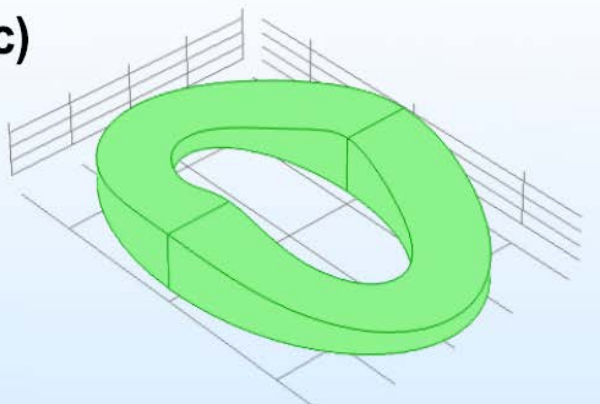

(b)

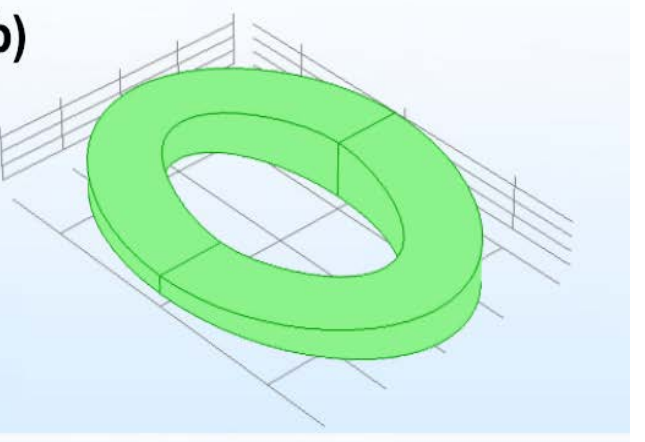

(d)

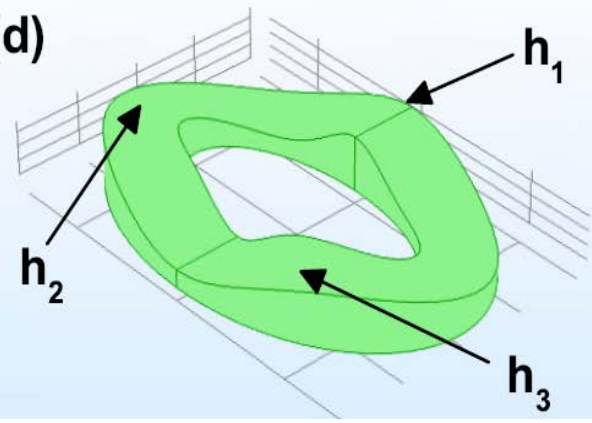

En la Figura 1, se muestran esquemas del sistema bajo estudio, modelado mediante el software COMSOL Multi-physics. En Figura 1(a), se muestra una representación del QR elíptico de GaAs sumergido en una matriz de AlGaAs, note que la sección transversal tiene una forma rectangular. En la Figura 1(b), se muestra la correspondiente malla construida para el sistema de la Figura 1(a); discretización del sistema que es usada para resolver la ecuación de Schrödinger mediante el MEF.

En la Figura 2 se muestran las diferentes configuraciones usadas en este trabajo, en donde se puede ver que el perfil de la sección transversal es rectangular. En los páneles cambia el número de puntos cuánticos de la siguiente manera: (a) $n=0$, (b) $n=1$, (c) $n=2$ y (d) $n=3$.

En la Figura 3 están indicadas las longitudes de la base de los QRs (plano xy). La longitud de los semiejes de la elipse interior sobre los eje $x$ y $y$ están dados por $R x_{1}$ y $R y_{1}$, respectivamente. La separación entre la elipse interior y exterior está dada por la constante $w p=10 \mathrm{~nm}$, esto es, la longitud de los semiejes de la elipse exterior son $R x_{2}=R x_{1}+w_{p}$ y $R y_{2}$ $=R y_{1}+w_{p}$.

Figura 3. Representación de la base de los QRs (plano xy). $R x_{1}$ y $R y_{1}$ son las longitudes de los semiejes de la elipse interior y wp es la separación entre la elipse interior y exterior.

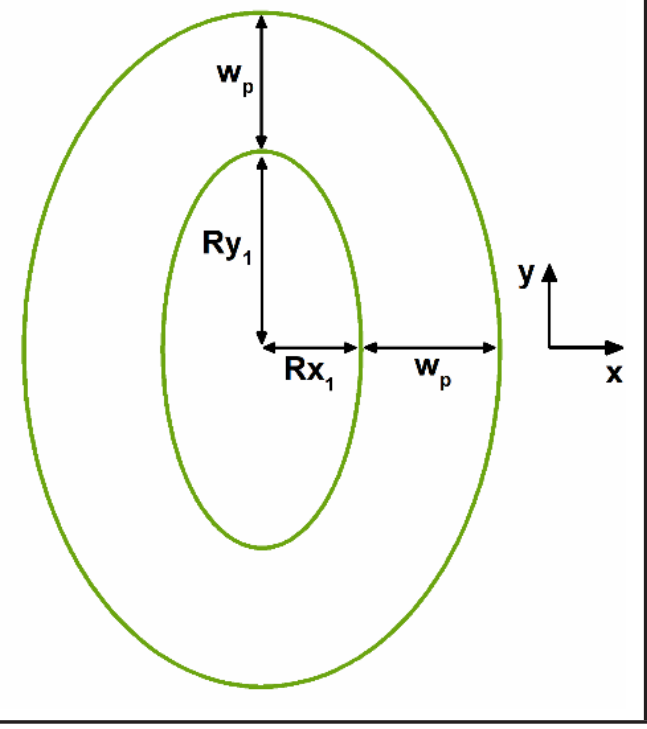


Figura 4. Proyección $z=2,5 \mathrm{~nm}$ de las primeras cinco funciones de onda de un electrón confinado en un QR elíptico para diferente número de puntos cuánticos, $n=0,1,2,3$ de la primera a la cuarta fila, respectivamente. Las elipses dibujadas en color negro corresponden a la base del $\mathrm{QR}$, esto es, $z=0$. Las dimensiones de la elipse interior son $R x_{1}=10 \mathrm{~nm}$ y $R y_{1}=20 \mathrm{~nm}$.
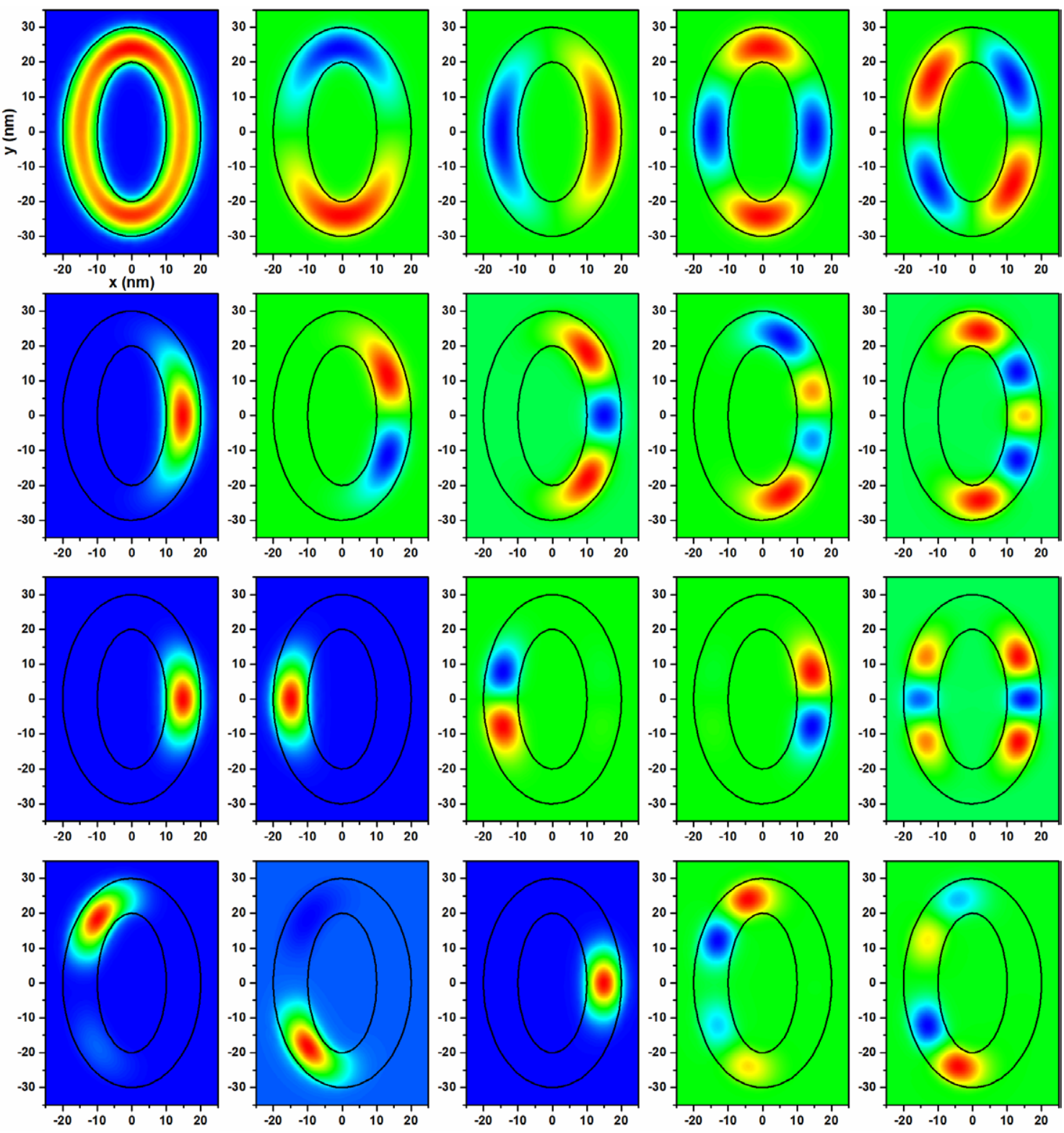

\section{Resultados y discusión}

En la Figura 4 se muestran las proyecciones $z=2,5 \mathrm{~nm}$ de las primeras cinco funciones de onda a través de las columnas y cuando se avanza a lo largo de las filas, tenemos diferente número de puntos cuánticos, de cero a tres $(n=0,1,2,3)$. En la primera fila [ $n=0$, ver Figura 2(a)], el estado fundamental tiene simetría tipo s, con mayor probabilidad de presencia del electrón sobre la zona de más confinamiento, esto es, en la parte superior e inferior de la elipse donde se tienen una mayor curvatura. El primer y segundo estado excitados tienen una simetría tipo p y son cuasidegenerados. 
El cuarto y quinto estado tienen simetría de inversión, con presencia del electrón sobre los extremos del eje $x$ y $y$. En la segunda fila $(n=1)$, en el que tenemos un punto cuántico como se ilustra en la Figura 2(b), vemos que las funciones de onda para los estados más bajos están concentradas sobre la posición del anillo donde aparece el punto cuántico, ya que es el lugar de menor confinamiento, por ser la región de mayor volumen. Conforme nos movemos hacia la derecha, las funciones de onda se extienden sobre el anillo, es decir, se acercan hacia zonas de menor altura. En la tercera fila $[n=2$, ver Figura 2(c)], donde tenemos dos puntos cuánticos, observamos que los primeros dos estados son degenerados y el electrón se localiza en las regiones de los puntos cuánticos. Los estados del tercero al quinto muestran que las funciones de onda son más extendidas alrededor de los puntos cuánticos. En la cuarta fila [ $n=3$, ver Figura 2(d)], para tres puntos cuánticos en el anillo, observamos que los primeros tres estados corresponden a la localización de los puntos cuánticos. El cuarto y quinto estado corresponden a funciones de onda con mayor densidad de probabilidad en los puntos cuánticos simétricos, es decir a lo largo del semieje mayor de la elipse.

En la Figura 5, se muestran las primeras energías de un electrón confinado en un QR elíptico como función de $R x_{1}$, con $R y_{1}=20 \mathrm{~nm}$. Diferente número de puntos cuánticos han sido considerados: (a) cero $(n=0)$, (b) uno $(n=1)$, (c) dos $(n=2)$ y (d) tres $(n=3)$. Los cálculos fueron realizados para $R y_{1}$ $=20 \mathrm{~nm}$. En la Figura 5(a), donde la altura del anillo es constante, en general se ve una disminución de las energías con el aumento de $R x_{1}$, ya que el volumen aumenta, lo que se traduce en un menor confinamiento. En los tres primeros niveles se observa un mínimo alrededor de $20 \mathrm{~nm}$ para los tres primeros niveles, lo cual es resultado de un cambio de confinamiento 3D a 2D, debido a que con el aumento de $R x_{1}$ a partir de $20 \mathrm{~nm}$ los extremos superior e inferior (viéndolos en un plano $x y$ ) del QR son cada vez más cercanos a ser paralelos, esto es, se pasa a un confinamiento 2D pues cuanto mayor será $R x_{1}$, estamos cerca de dos hilos cuánticos paralelos. En la Figura 5(b), donde se tiene un solo punto cuántico (como en la Figura 2(b)), tenemos una asimetría, en los extremos del anillo, de ahí que los niveles de energía no estén degenerados y se trate de mayores valores de energía ya que el electrón está confinado en una región más pequeña. En la Figura 5(c), al tener dos puntos cuánticos en los extremos (como en la Figura 2(c)), los estados tienen degeneración doble, lo cual es señalado por el número 2 en la figura y es resultado de tener dos puntos cuánticos idénticos y casi aislados. En la Figura 5(d), en donde hay un número impar de puntos cuánticos $(n=3$ como en la Figura 2(d)), están etiquetados los niveles de acuerdo con la degeneración, 1 para no degenerados y 2 para doblemente degenerados. Para elipticidades positivas $\left(R x_{1}>20 \mathrm{~nm}\right)$ el electrón tiene el siguiente comportamiento: El punto cuántico etiquetado como $\mathrm{h}_{1}$ Figura 2(d) contiene el estado fundamental y vemos que no es degenerado, ya que este punto cuántico es diferente a los puntos cuánticos etiquetados como $\mathrm{h}_{2} \mathrm{y} \mathrm{h}_{3}$. El segundo y tercer nivel son cuasidegenerados debido a que los puntos cuánticos etiquetados como $\mathrm{h}_{2} \mathrm{y} \mathrm{h}_{3}$ tienen una región idéntica, este comportamiento es análogo para niveles superiores.

En cuanto a los QR elípticos para elipticidad negativa $\left(R x_{1}<20 \mathrm{~nm}\right)$, se observa que el estado fundamental es degenerado debido a que corresponde a los puntos cuánticos idénticos $\mathrm{h}_{2} \mathrm{y} \mathrm{h}_{3}$, el tercer estado corresponde al punto cuántico $\mathrm{h}_{1}$, el cual es diferente a los otros dos, de ahí que no se presente degeneración. Es decir, con el cambio de signo en la elipticidad tenemos una inversión entre los niveles. En la línea punteada vertical en $R x_{1}=20 \mathrm{~nm}$ el cual corresponde a un $\mathrm{QR}$ circular se tienen tres puntos cuánticos idénticos, de ahí que en los primeros 9 niveles de energía se tenga una degeneración triple. En los últimos estados mostrados en la figura, esto es, del 10 al 12 se pierde la degeneración debido a que las funciones de onda son más extendidas, así que se pierde el aislamiento de los puntos cuánticos. 
Figura 5. Niveles de energía de un electrón confinado en un QR elíptico como función de $R x_{1}$, con $R y_{1}=20 \mathrm{~nm}$. Diferente número de puntos cuánticos han sido considerados: (a) $n=0$ correspondiente a una altura constante del anillo como en la Figura 2(a), (b) $n=1$ un punto cuántico como en la Figura 2(b), (c) $n=2$ dos puntos cuánticos como en la Figura 2(c) y (d) $n=3$ tres puntos cuánticos como en la Figura $2(\mathrm{~d})$.
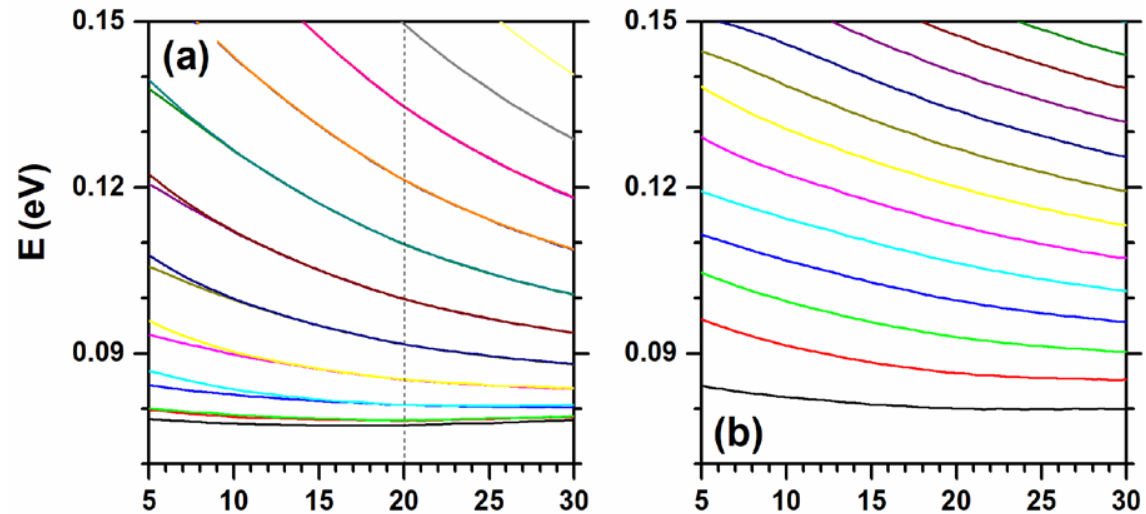

$\operatorname{Rx}_{1}(\mathrm{~nm})$
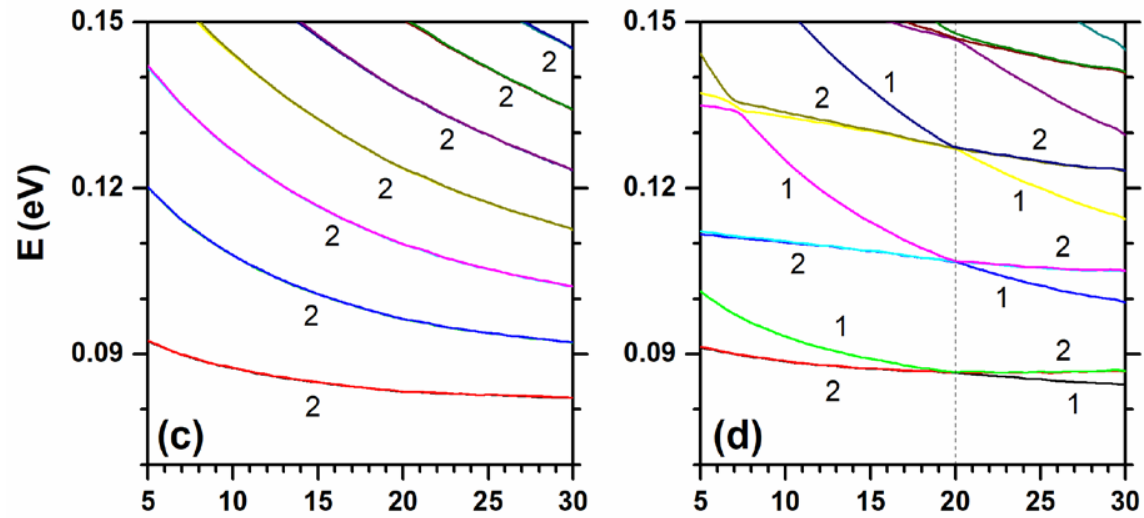

$\operatorname{Rx}_{1}(\mathrm{~nm})$

En la Figura 6 se muestran las primeras energías para un electrón confinado en un QR elíptico como función de la amplitud $A$ (ver Ecuación 4). En la Figura 6(a), las energías muestran una degeneración doble, lo cual está señalado por el número 2 en la figura, este comportamiento incluye al estado fundamental que presenta degeneración doble (excepto para amplitudes pequeñas), lo cual es resultado del aislamiento entre los puntos. Esta doble degeneración alcanza a ser visible para un número mayor de estados superiores conforme nos movemos a valores más altos de $\mathrm{A}$, ya que el aislamiento entre los QDs se ve aumentado a medida que crece dicho parámetro $A$. En la Figura 6(b), se muestran las primeras nueve energías para valores pequeños de la amplitud $A$. Para valores de $A$ cercanos a cero, vemos que el estado fundamental no presenta cuasidegeneración y los estados superiores presentan cuasidegeneración doble, condición que corresponde a lo observado para QRs de altura constante en la Figura 5(a). A partir de $A=0.2 \mathrm{~nm}$ se vuelven apreciables los efectos de tener dos QDs para el estado fundamental, ya que aparece una cuasidegeneración, lo propio ocurrirá para los estados de mayor energía, que alcanzarán cuasidegeneración doble para valores cada vez mayores de $A$, lo cual está en concordancia con la Figura 5(c) para dos QDs. 
Figura 6. Niveles de energía de un electrón confinado en un QR elíptico como función de la amplitud $A$ [ver Ecuación (4)], para dos puntos cuánticos opuestos $[n=2$, ver Figura 2(c)]. Las dimensiones de la elipse interna son $R x_{1}=10 \mathrm{~nm}$ y $R y_{1}=20 \mathrm{~nm}$.

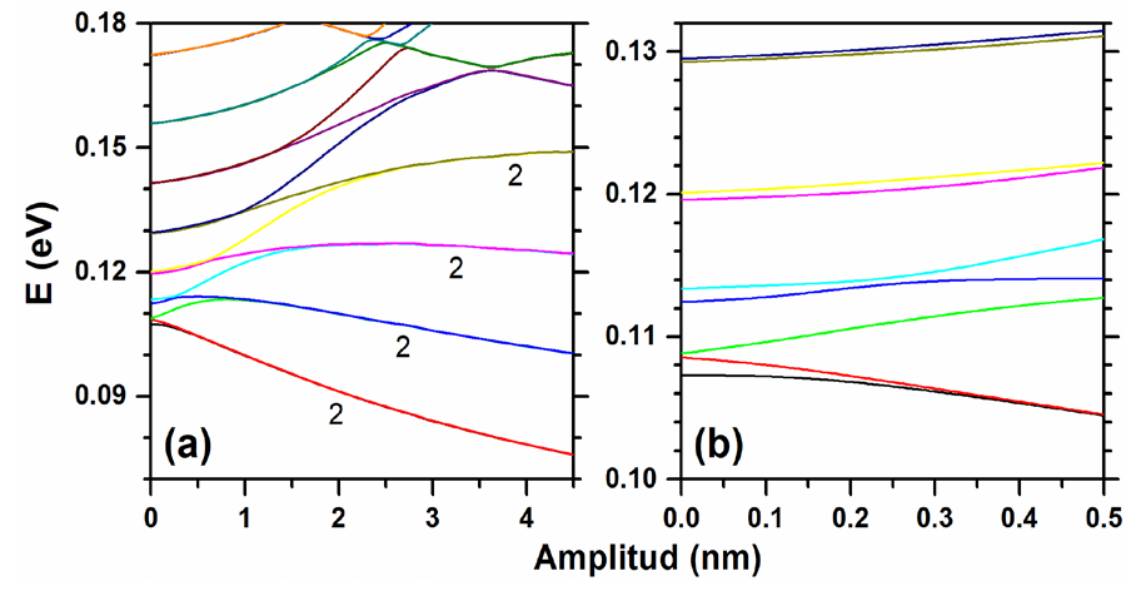

\section{Conclusiones}

En este artículo se ha estudiado el espectro energético para un electrón confinado en un QR elíptico con sección transversal rectangular, variando sus dimensiones y modulando la altura en dirección angular, lo que permite la obtención de diferente número de puntos cuánticos sobre el QR. Adicionalmente, se varía la amplitud de modulación de la altura por medio de funciones trigonométricas. La ecuación de Schrödinger 3D ha sido resuelta mediante el método de elementos finitos.

Para los primeros estados, el electrón tiene una localización sobre las regiones de mayor volumen. En el caso de los QRs con puntos cuánticos, la localización está sobre los puntos cuánticos de mayor volumen. Para los estados de mayor energía las funciones de onda tienen una mayor extensión sobre el QR, indicando presencia del electrón sobre zonas de mayor confinamiento. Hay aparición de cuasidegeraciones o pérdida de estas dependiendo de un número par $(n=0,2)$ o impar $(n=1,3)$ de puntos cuánticos. Con relación a la amplitud de modulación en la altura del QR, es claro con los resultados obtenidos, el aislamiento de los puntos cuánticos aún para valores pequeños de la amplitud $(A>0,2 \mathrm{~nm})$.

\section{Agradecimientos}

Esta investigación fue realizada gracias al apoyo de las instituciones colombianas: CODIUniversidad de Antioquia (Estrategia de Sostenibilidad de la Universidad de Antioquia y proyecto "Efectos de capas delta dopadas en pozos cuánticos como fotodetectores en el infrarrojo") y la Facultad de Ciencias Exactas y Naturales-Universidad de Antioquia (CAD- proyecto de dedicación exclusiva 2017-2018). J.A.V agradece el apoyo financiero para su formación doctoral por parte de la Agencia Colombiana El Patrimonio Autónomo Fondo Nacional de Financiamiento para la Ciencia, la Tecnología y la Innovación, Francisco José de Caldas- COLCIENCIAS.

\section{Referencias}

Aharonov, Y.; Bohm, D. (1959). Significance of Electromagnetic Potentials in the Quantum Theory. Phys. Rev., 115(3), pp. 485-491.

Baker, C.; Lo, T.; Tribe, W. R.; Cole, B. E.; Hogbin, M. R.; Kemp, M. C. (2007). Detection of Concealed Explosives at a Distance Using Terahertz Technology. Proceedings of the IEEE, 95(8), pp. 1559-1565.

Bejan, D.; Stan, C.; Niculescu, E. C. (2018). Effects of electric field and light polarization on the electromagnetically induced transparency in an impurity doped quantum ring. Optical Materials, 75, pp. 827-840. 
Bejan, D.; Stan, C.; Niculescu, E. C. (2018). Optical properties of an elliptic quantum ring: Eccentricity and electric field effects. Optical Materials, 78, pp. 207-219.

Boonpeng, P.; Kiravittaya, S.; Thainoi, S.; Panyakeow, S.; Ratanathammaphan, S. (2013). InGaAs quantumdot-in-ring structure by droplet epitaxy. J. Crystal Growth, 378, pp. 435-438.

Büttiker, M.; Imry, Y.; Landauer, R. (1983). Josephson behavior in small normal one-dimensional rings. Phys. Lett. A, 96(7), pp. 365-367.

Chakraborty, T.; Manaselyan, A.; Barseghyan, M.; Laroze, D. (2018). Controllable continuous evolution of electronic states in a single quantum ring. Phys. Rev. B, 97(4), pp. 41304.

Cheng, K. A.; Yang, C. H.; Yang, M. J. (2000). Nanometersize InAs/AlSb quantum wires: Fabrication and characterization of Aharonov-Bohm quantum rings. J. Appl. Phys., 88(9), pp. 5272-5276.

Collier, T. P.; Saroka, V. A.; Portnoi, M. E. (2017). Tuning terahertz transitions in a double-gated quantum ring. Phys. Rev. B, 96(23), pp. 235430.

COMSOL Multiphysics, v. 5.3a. COMSOL AB, Stockholm, Sweden.

El-Bakkari, K.; Sali, A.; Iqraoun, E.; Rezzouk, A.; Es-Sbai, N.; Jamil, M. O. (2018). Effects of the temperature and pressure on the electronic and optical properties of an exciton in GaAs/ $\mathrm{Ga}_{1-\mathrm{x}} \mathrm{Al}_{\mathrm{x}}$ As quantum ring. Physica B, 538, pp. 85-94.

Escorcia, R.; García, L. F.; Mikhailov, I. D. (2018). Magnetoelectric effect in concentric quantum rings induced by shallow donor. Physica E, 99, pp. 269-274.

Fomin, V. M. (2014). Physics of Quantum Rings, SpringerVerlag, Berlin, pp. 27-193.

Gómez, C. A.; Marín, J. H.; Gutiérrez, W.; García, L. F. (2009). Denergy spectrum in toroidal quantum ring. J. Phys.: Conference Series, 167(1), pp. 12032.

He, Z.-L.; Bai, J.-Y.; Ye, S.-J.; Li, L.; Li, C.-X. (2017). Quantum Switch and Efficient Spin-Filter in a System Consisting of Multiple Three-Quantum-Dot Rings. Chinese Phys. Lett., 34(8), pp. 87301.

Hu, M.; Wang, H.; Gong, Q.; Wang, S. (2018). External electric field effect on the binding energy of a hydrogenic donor impurity in InGaAsP/InP concentric double quantum rings. International Journal of Modern Physics B, 32(11), pp. 1850138.
Kuroda, T.; Mano, T.; Ochiai, T.; Sanguinetti, S.; Sakoda, K.; Kido, G.; Koguchi, N. (2005). Optical transitions in quantum ring complexes. Phys. Rev. B, 72(20), pp. 205301.

Lee, C. M.; Li, J. Q.; Ruan, W. Y.; Lee, R. C. H. (2006). Optical spectra and intensities of a magnetic quantum ring bound to an off-center neutral donor $\mathrm{D}^{0}$. Phys. Rev. B, 73(21), pp. 212407.

Linares-García, G.; Meza-Montes, L.; Stinaff, E.; Alsolamy, S. M.; Ware, M. E.; Mazur, Y. I.; Wang, Z. M.; Lee, J.; Salamo, G. J. (2016). Optical Properties of a Quantum Dot-Ring System Grown Using Droplet Epitaxy. Nanoscale Res. Lett., 11(1), pp. 309.

Ling, H. S.; Wang, S. Y.; Lee, C. P.; Lo, M. C. (2009). Characteristics of $\operatorname{In}(\mathrm{Ga})$ As quantum ring infrared photodetectors. J. Appl. Phys., 105(3), pp. 34504.

Ling, H.-S.; Lee, C.-P. (2007). Evolution of self-assembled InAs quantum ring formation. J. Appl. Phys., 102(2), pp. 24314.

Lorke, A; Garcia, J. M.; Blossey, R.; Luyken, R. J.; Petroff, P. M. (2003). Self-Organized InGaAs Quantum Rings Fabrication and Spectroscopy. In B. Kramer (Ed.), Advances in Solid State Physics 43, pp. 125.

Lorke, A.; Johannes Luyken, R.; Govorov, A. O.; Kotthaus, J. P.; Garcia, J. M.; Petroff, P. M. (2000). Spectroscopy of Nanoscopic Semiconductor Rings. Phys. Rev. Lett., 84(10), pp. 2223-2226.

Mano, T.; Kuroda, T.; Mitsuishi, K.; Yamagiwa, M.; Guo, X.-J.; Furuya, K.; Sakoda, K.; Koguchi, N. (2007). Ring-shaped GaAs quantum dot laser grown by droplet epitaxy: Effects of post-growth annealing on structural and optical properties. J. Crystal Growth, 301-302, pp. 740-743.

Mughnetsyan, V. N.; Manaselyan, A. K.; Barseghyan, M. G.; Kirakosyan, A. A. (2013). Simultaneous effects of hydrostatic pressure and spin-orbit coupling on linear and nonlinear intraband optical absorption coefficients in a GaAs quantum ring. J. Lumin., 134, pp. 24-27.

Mughnetsyan, V.; Kirakosyan, A. (2017). Strain distribution and band structure of InAs/GaAs quantum ring superlattice. Superlattice Microstruct., 112, pp. 318-327.

Rosas, R.; Riera, R.; Marín, J. L. (2000). Electron states in a magnetic quantum ring. J. Phys.: Condensed Matter, 12(30), pp. 6851. 
Shi, L.; Yan, Z. W. (2018). Stark shift and photoionization cross section of on-center and off-center donor impurity in a core/shell ellipsoidal quantum dot. Physica E, 98, pp. 111-117.

Vinasco, J. A.; Londoño, M. A.; Restrepo, R. L.; Mora-Ramos, M. E.; Feddi, E. M.; Radu, A.; Kasapoglu, E.; Morales, A. L.; Duque, C. A. (2017). Optical Absorption and Electroabsorption Related to Electronic and Single Dopant Transitions in Holey Elliptical GaAs Quantum Dots. Physica Status Solidi B, 255(4), pp. 1700470.

Wu, J.; Wang, Z. M.; Holmes, K.; Marega, E.; Zhou, Z.; Li, H.; Mazur, Y. I; Salamo, G. J. (2012). Laterally aligned quantum rings: From one-dimensional chains to two-dimensional arrays. Appl. Phys. Lett., 100(20), pp. 203117.

Zamani, A.; Setareh, F.; Azargoshasb, T.; Niknam, E. (2018). Spin-orbit coupling and applied magnetic field effects on electromagnetically induced transparency of a quantum ring at finite temperature. Superlattice Microstruct., 115, pp. 40-52.

\section{PARA CITAR ESTE ARTÍCULO / TO REFERENCE THIS ARTICLE / PARA CITAR ESTE ARTIGO /}

Vinasco Suárez, J.A.; Radu, A.; Duque Echeverri, C.A. (2019). Propiedades electrónicas de un anillo cuántico elíptico con sección transversal rectangular (Anillo cuántico elíptico). Revista EIA, 16(31), enero-junio, pp. 77-87. [Online]. Disponible en: https://doi.org/10.24050/reia.v16i31.1255 